\title{
HYBRID SIMULATION NETWORK FOR VEHICULAR AD HOC NETWORK (VANET)
}

\author{
Chun Hoe Lee, Kit Guan Lim, Min Keng Tan, Renee Ka Yin Chin and Kenneth Tze Kin Teo \\ Modelling, Simulation and Computing Laboratory, Universiti Malaysia Sabah, Malaysia
}

\begin{abstract}
Intelligent Transportation Systems (ITS) plays a vital role in providing different means of traffic management and enables users to be better informed of traffic condition, promoting safer, coordinated and efficient use of transport network. Vehicular Ad Hoc Network (VANET) shows promising reliability and validity in ITS. But, it poses challenges to researchers in designing protocol specifically for VANET as the deployment of VANET in real world will incur high cost. Therefore, simulation and non-physical testbed implementation have been widely adopted by the VANET research community in the development and assessment of the new or improved system and protocol of VANET. This paper presents a viable simulation platform for network development. Besides, a code cast or better known as network coding, a data packet transmission method has been developed and introduced into VANET protocol using the presented platform to assess and determine the potential of the introduced simulation platform.
\end{abstract}

\section{Keywords:}

Vehicular Ad hoc Network, Traffic Simulation, Network Simulation, Intelligent Transportation System, Network Coding

\section{INTRODUCTION}

Communication becomes ever more important as it has a direct impact on daily productivity. Hence, in modern days, field of communication is a popular subjects for various researchers and developers especially the field related to the intelligent transportation system (ITS). Most of these traffic accidents and car collisions are avoidable or mitigate if using ITS and safety communication related technology because driver will be wellinformed about any potential accident and collision ahead by giving a collision warning, emergency electronic brake lights and slow or halt vehicles. Besides, alerts for information such as sharp velocity changes in vehicles ahead is also able to alleviate accidents and collisions.

Several systems have been developed in such a way that the driver can estimate traffic conditions and travel time using smartphone. The system is better known as cooperative driver assistance. However, the accuracy and precision of the information reported are highly debatable as the information is the input updated by publics and information contributed might not up-to-date. The creation of the ITS holds a huge benefits such as road safety, traveller comfort and traffic efficiency. Generally, ITS upholds three main notions which are the advanced driver assistance, resources efficiency such as traffic and vehicle, and safety [1]. One of the many strategies for creating a safer and less congested road has been the development of ITS, the incorporation of information and communication technologies in vehicles and transport infrastructure called Vehicular Ad Hoc Network (VANET).

VANET has emerged as a very promising technology ever since the ITS is implemented [2]. It is a subset of mobile ad hoc networks (MANET). Similar to MANET, VANET does not rely on fixed infrastructure to discriminate data but rather depend on the ordinary nodes to perform routing of message with its own networking management [3] - [6]. The technology of VANET has evolved from MANET to support vehicle-to-vehicle, vehicle-toinfrastructure and vehicle-to-people communication over the past few years. In a VANET, high mobility vehicles and road side units are the nodes that communicate with each other for information sharing. Each participating vehicle will acts as a wireless router that establishes the connection or bridges the link, allowing nearby vehicle to connect with one another, or a data source to gather the information from the sensors such as speed detector and camera found on the roadside and transmit it to all the nearby cars, and a data destination to receive and display the traffic relevant information for motorist.

Current researches are mainly focus on the operation and management of communication networks in VANET in terms of reliability, security, robustness and throughput [4]. There are many benefits bring about by VANET application, but people cannot benefit from it yet. There are also a number of hindrances in VANET required an immediate, effective and reactive measure from researchers to solve the problems related to high connectivity and bandwidth, security and privacy and finally, provision of quality of service (QoS) [3].

Besides, the performance modelling of VANET is crucial in determining its success of development and deployment in this research. The development of packet transmission and routing protocol require accurate model of VANET in order to reflect accurately the behaviour of the protocol in the real world. Hence, the successful modelling of VANET is highly depending on the mobility model and its real world environment.

Generally, VANET simulation comprises two types of simulation components which are network and traffic mobility simulation. There are several network simulators available for deployment such as NS2 and OMNET that can be used to generate a platform to test and evaluate the network protocols. These tools are designed to provide generic simulation network scenario without being particular tailored to support varieties applications of VANET such as collision avoidance, road obstacle warning, traffic information and other non-safety services. Network generator cannot generate the movement of the node but is able to develop communication protocol that allows the node to communicate with one another within the ad hoc network.

On the other hand, traffic mobility simulation is used to describe the movement and behaviour of the vehicles on a network restricted by the road layout, other vehicle's movement, road obstacles and traffic signal at the junction. Coupling between network and traffic mobility simulators allow different vehicular scenarios to be produced and the characteristic of radio propagation between the vehicles and on-site unit on different generated environments to be observed and evaluated. 
So, this paper generates a realistic road topologies as close as possible to real-world behaviour in order to ensure the simulation results accurately reflect the performance of VANET in realworld using traffic simulator. Different set of transmission protocols have been developed and simulated to determine accessibility and viability of the suggested simulation platform.

\section{TRAFFIC SIMULATION}

Simulation of wireless ad hoc network requires continuous monitoring of node positions at any given instant, with no exception for vehicular networks. Mobility movement of vehicular network is unique as the nodes in the network move along the roads in a predefined direction compared to mobile ad hoc network (MANET) where its nodes can move freely in any direction. The continuous tracking of node movement is vital as the location of the nodes in the topology at any instant is directly influencing the network connectivity and packet delivery. A large number of traffic variables such as (section 2.1) street layout, (section 2.2) traffic signal and (section 2.3) obstacles and vehicular behaviour are mostly not considered in the conventional mobility models. These factors that influence the mobility in VANET simulation are described as following.

\subsection{STREET LAYOUT}

The road layouts confine the movements of each node to welldefined paths regardless of its final destination. This constrained movement pattern defines the distribution of nodes and their connectivity.

\subsection{TRAFFIC SIGNAL}

The stop sign and traffic lights in the intersection result in queues of vehicles at the intersection. These queues in the intersection implies more static nodes with slower rate of transmission route changes in network and better network connectivity. However, it can also adversely affect network performance with increased transmission delay and channel utilisation.

\subsection{ROAD SIGN AND VEHICULAR BEHAVIOUR}

Speed bump and speed limit sign are examples of the obstacle that impede the flow of vehicles in a topology. Vehicular movement in VANET also can be influenced by the movement of other vehicles surrounding it. For instance, a vehicle would maintain a minimum distance from the vehicle in front of it, by accelerate, decelerate or change to another lane to avoid collision.

As a result of ignorance of these features in VANET simulation leads to infeasible simulation results and unable to predict the developed protocol performance in the real world accurately. Therefore, the topologies for VANET have to be modelled and simulated as close as possible to real-world behaviour in order to ensure the simulation results accurately reflect the performance of VANET in real-world, at the same time ease the deployment of the developed model or protocol in the near future.

Traffic simulation can be classified into three models namely the microscopic, mesoscopic and macroscopic model [7]. Macroscopic model deals with traffic flow, traffic density, and the distribution of vehicles. While microscopic approach describes the movement of each individual vehicle and its behaviour on the road. Last but not least, mesoscopic model is employed as an intermediate approach to aggregate the movement of the nodes. In order to generate a realistic urban scenario, microscopic model is used to customize vehicles scenario, road obstacles and car's parameters, such as car speed, arrival and departure time of each node. There are several traffic mobility candidates that are able to generate all the above-mentioned characteristics such as Simulation of Urban Mobility or SUMO, VANETSim and CanuMobiSim [8]-[10]. SUMO and VANETSim have been designed to generate transportation scenario at microscopic and macroscopic level, which is beneficial for it to be used in evaluating VANET protocol modelled by the researchers in later part of their research.

Among these traffic mobility simulators, SUMO able to handle large road network in microscopic level and compatible with several of commercially available data simulators. SUMO is also able to generate traffic demands including vehicle's travelling details such as speed, direction and position along the time. Therefore, ultimately, SUMO is used to generate traffic information in this research work.

Sumo is an open source, multi-modal traffic simulator developed by German Aerospace Centre, Institute of Transportation System. It allows the implementation of custom traffic scenario, including road network and traffic demand. Road network defines the roads layout, streets, traffic signal and junction, while traffic demand describes the behaviour of each vehicle. SUMO can be used to deal with a large set of traffic management, while in microscopic level it can model each vehicle with individual characteristics like the vehicles movement throughout the network. Simulation with SUMO is deterministic in nature but the randomness can be introduced to the simulation. SUMO offers multiple ways to generate a road map, either import directly from Open Street Map (OSM) or create manually by the user, using NETGENERATOR function provided by SUMO. In addition, it can be generated automatically through some other intermediate framework like Mobility Model Generator for Vehicular Networks (MOVE). The map imported from OSM server comes with buildings, traffic signals, turn restriction and other traffic obstacles which make the generated topology a more realistic environment as shown in Fig.1. The imported map can be edited using JOSM, an extensive, open source editor for OSM which is written in Java language before converted into SUMO readable format using NETCONVERT function found in SUMO.

Google Earth has been integrated into MOVE to facilitate the creation of node in a realistic setting. The latitude and longitude information of selected locations can be set and saved into a file in kml format. Besides, a well-known Topologically Integrated Geographic Encoding and Referencing (TIGER) database can also be imported using MOVE framework. TIGER is a format used by the United State Census Board to describe the land attributes such as road and buildings for public domain. Vehicle Movement Editor incorporated in MOVE allows user to create the route, trip, pattern and direction of each vehicle either manually defined by the user or automatically generated. SUMO then generates the mobility trace which is used by network simulator such as OMNET to simulate the realistic vehicle movement with the input information defined in MOVE. MOVE allows users to 
easily generated real world mobility model for VANET simulation but SUMO itself suffices to simulate the realistic traffic simulation as required for this research work.

SUMO can generate the vehicle's physical properties automatically using randomtrips.py, a script function found in SUMO package. Traffic demand and route data with vehicle's properties are defined together in a file and saved in rou.xml format. The traffic demand defined in rou.xml comprises of four attributes including the departure time of specific vehicle, id of specific vehicle which is defined by the user, route used by the defined vehicle and type of vehicle. The realistic map from OSM together with traffic demand files generated are then compiled and called in a configuration file. The Fig. 2 shows the simulation of traffic mobility using SUMO.

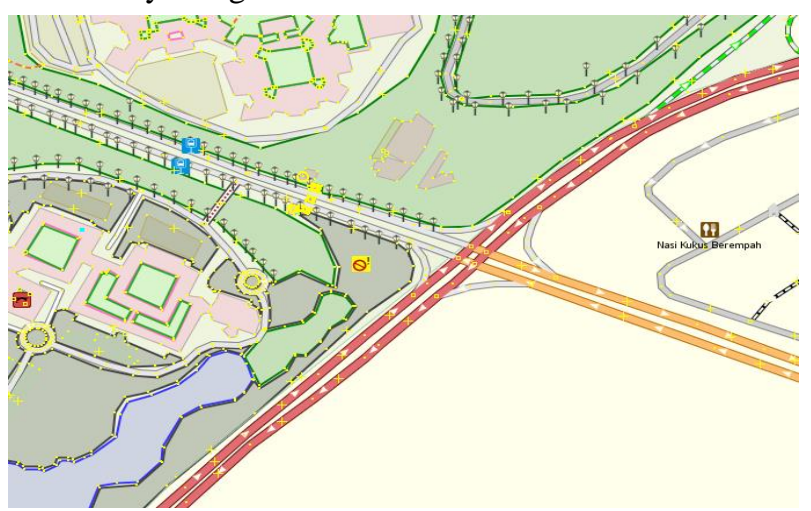

Fig.1. Map of Kota Kinabalu, Sabah, Malaysia from Open Street Map

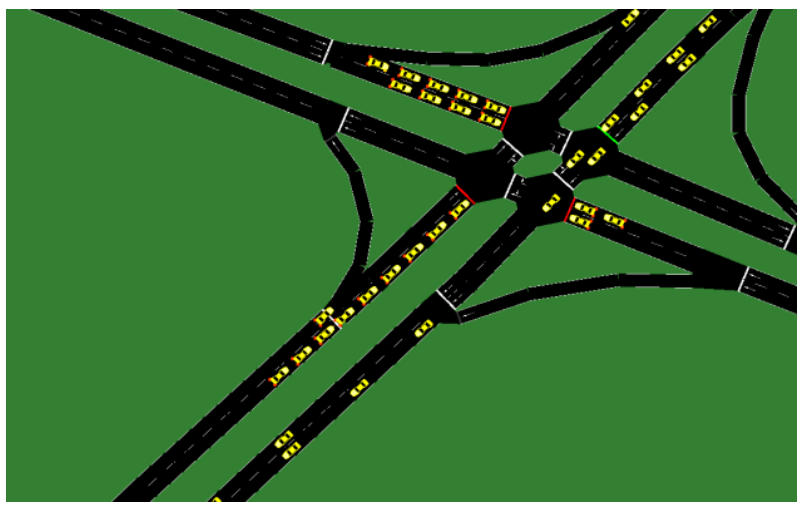

Fig.2. Traffic simulation using SUMO

\section{NETWORK SIMULATION}

Multihop ad hoc network paradigm is considered to be the backbone for many VANET development. Besides, the researches on VANET are mostly inherited from the result of MANET related to multihop ad hoc transmission and routing protocol which have been modified and adjusted to acclimate to the feature of vehicular field. It is critical that a proper network modelling algorithm must be used in ad hoc network simulation to yield a realistic result. There are several open source network simulators available for utilization such as NS2, NS3, Matlab and OMNET. In this paper, transmission protocol in VANET is simulated and developed by using Matlab-m by referring to the Open System Interconnection (OSI) layer and Wireless Access in a Vehicular Environment (WAVE) model which is defined by IEEE 802 group.

OSI model is a conceptual model of communication used to define and standardise the communication protocol in information transmission. It is often referred as a guideline in developing communication protocol [10]. OSI protocol is divided into seven layers namely the physical layer, data link layer, network layer, transport layer, session layer, presentation layer and application layer. Each layer is self-reliant, so it does not affect other layers if a single layer is altered or updated with another solution. Each layer has its own specific functions or task in packet handling within the communication node. The process of dividing functions or task into layers is important to reduce the complexity of networking.

During the packet transmission, the packet flows down through each layer of OSI in the source node, from the top application layer to the bottom physical layer. Then it is passed to the receiving node from the bottom physical layer to top application layer of receiving node. The Fig.3 shows the OSI model packet flow.

OSI layered model is only used as a reference model. It helps to breakdown problem involved in moving packet from one node to another node by categorising it into seven layers. Actual communication protocol stacks often combine one or more OSI layers in a single layer. It can be grouped into two sub layers: the upper layers and lower layers. The upper layers compose of the application, presentation, session and transport layer of OSI model. Whilst, the lower layers include the network, data link and physical layer.

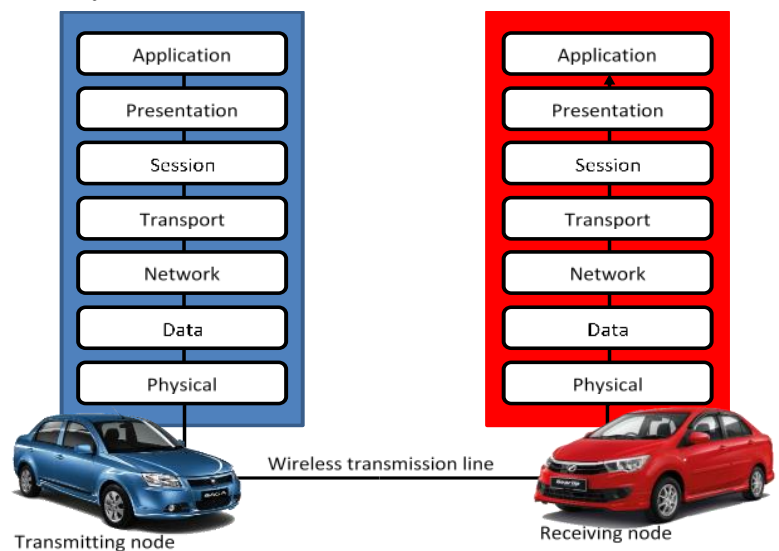

Fig.3. Process of communication between nodes

Generally, the upper layers deal with the protocol that enables the human network to interface with the underlying data network, for instance, web browser in computer. While, the lower layers are primarily concerned with the details of packet transmission over the network including the formatting and encoding of packet. The Fig.4 illustrates the division between the upper and lower layers of OSI.

The lower layer of the OSI model starts with the physical layer. It provides an overlay for the electrical signal and the physical specifications for a node. It physically controls the transmission of actual packet in the form of electrical impulse, light or radio signal trough the network at the electrical or optical and mechanical level. In other words, the lower layer is 
responsible for the connection between the nodes by physical means of sending and receiving data, including the wired cable, fibre optic and electromagnetic wave.

All the data transmitted over the physical layer are encoded in a sequence of bits which is also known as bit stream in the form of binary digit. These digit are most commonly represented as either 0 or 1 . But these values will be formatted into frame at the data link layer. It can be visualised as the bit stream is being arranged into an envelope with the envelope contains both sender and receiver's address information [11, 12]. Link establishment and termination between two nodes is controlled by the data link layer. In order to provides an error-free transfer of data frames from one node to another over the physical layer, each transmitter will send a Request-to-Send (RTS) packet which is also known as frame acknowledgements to the respective receivers to determine the availability of the receiver nodes before the real packet is being transferred from the transmitter. If the receiver is in idle mode, then the Clear-to-Send (CTS) packet will be replied to the transmitter so that the transmitter knows it can send the packet to the idle receiver. These phenomenon are solely controlled by data link layer.

The network layer of the OSI model is a network controller that manages the subnet traffic. It is a layer above the data link layer. It decides the end-to-end delivery of the packets based on the network conditions and priority of services. It also defines the process used to format packet into a smaller packet in order to accommodate different type of message or media. Ultimately, it controls the routing of the packet transmission and ensure the delivery of the packet in the right direction and the right hop.

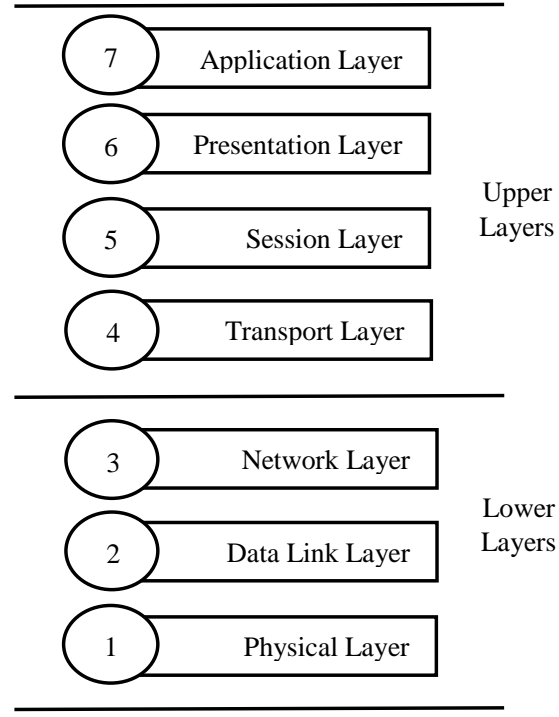

Fig.4. Open systems interconnection model

The transport layer is the fourth layer in the OSI model that regulates the information flow to ensure the reliability and security of the host application or message in the end-to-end connectivity. This layer responsible for the disassembly and assembly of the data before and after transmission. The session layer establishes and maintains the session between the nodes. It defines the setup, control and terminates conversations, exchanges and dialogues between the applications at each end. Meanwhile, the presentation layer is concerned with data representation where the format of the data will be translated, compressed and encrypted before presented to the next layer. It ensures the information send out is readable by translating from network to application format and vice versa. Finally, the application layer is the seventh layer and uppermost layer in the OSI model which is the closest layer to the end user. It is different from other OSI layers as it does not provide any services to other OSI layer. Instead, it only works towards the application outside the model.

The Institute of Electrical and Electronics Engineers Standards Association (IEEE-SA) is an organization within IEEE that develops global standard in various industrials with the aim to nurture, develop and advance global technologies. The IEEE develops its standard through a consensus development process, involving the volunteers representing varied viewpoints and interest in the related field. Despite the vast research efforts, VANET has limited role in ITS. This is mainly due to a lack of realism in the research objectives with lack of adherence to the modelling guidelines. Hence, the IEEE group has defined the first version of the protocol stack IEEE 802.11p and IEEE 1609.x specifically to adapt vehicular environment [13] - [15]. Both IEEE 802.11p and IEEE 1609 are part of the wireless access in a vehicular environment (WAVE) architecture. The WAVE architecture utilises the dedicated short-range communication (DSRC) spectrum band, also recognised as intelligent systems radio service to establish communication between high speed vehicles and between the vehicles and the roadside infrastructure.

DSRC has been introduced to support a plethora applications of vehicular communication such as collision avoidance and road obstacle warning. The Federal Communications Commission (FCC) has allocated $75 \mathrm{MHz}$ of spectrum at $5.9 \mathrm{GHz}$ specifically for DSRC in USA to provide public safety applications and improve traffic efficiency. The bandwidth is structured into seven frequency channels with $10 \mathrm{MHz}$ wide each as shown in Fig.5.

The inclusion of OSI layer as well as the implementation of IEEE 802 architecture at the physical layer is necessary to properly establish a consistent protocol that can be universally adopted for the usage of VANET research. The specification and procedures designed is vital to ensure VANET functionality, facilitate interoperability and reliability for everyday use.

Channel 178 is the control channel $(\mathrm{CCH})$, which is reserved for safety message (beacon) and for system control and management purposes. Meanwhile, the remaining channels are the service channel (SCHs) used to support for non-safety usage relevant applications. The two channels at both ends of the spectrum band are kept for special uses. Application related to VANET are generally classified into two categories namely safety applications and non-safety applications. Safety application provide driver information about the critical situation in advance such as speed of the vehicles ahead and road condition to the drivers, allowing the drivers to have adequate time to react upon the information received and eliminating the sudden braking or stop which are believed to be major cause of traffic casualties. On the other hand, non-safety application are used to provide drivers information with related to traffic efficiency and entertainment. These are meant for improving driving comfort and driving experience. Other non-safety applications including business advertisement and general information services that are envisioned in the application of VANET such as cooperative adaptive cruise control, speed limit warning, animal warning, map 
and media update and so on. In Malaysia, Malaysian Communications and Multimedia Commission (MCMC) does not specific provide band for vehicular communication but allocates several bands for the usage of Industrial, Scientific and Medical (ISM) purpose.

IEEE $802.11 \mathrm{p}$ standard defines the physical and medium access control layers, where necessary functions and services which are required by the WAVE stations including the participating vehicles that are moving in rapid changing environment. WAVE protocol introduced also exhibits similar properties of OSI layers as shown in Fig.6.

Medium access control (MAC) architecture in the WAVE model is responsible for channel routing, channel coordination and prioritization using backoff and timing. The behaviour of the wireless transmission in real world where the interference, reception model and radio propagation model will be implemented in the physical (PHY) layer. Meanwhile, logical link control (LLC) sublayer is used to provide multiplexing mechanism when transmitting, and decoding when receiving message.

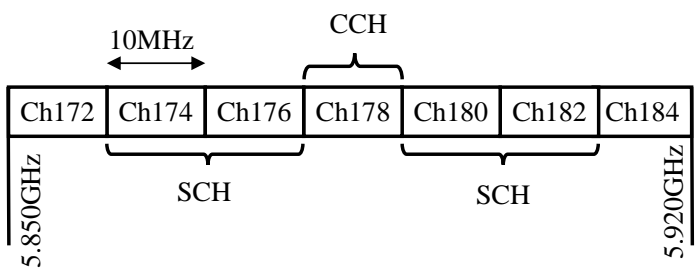

Fig.5. WAVE Radio Channel Management

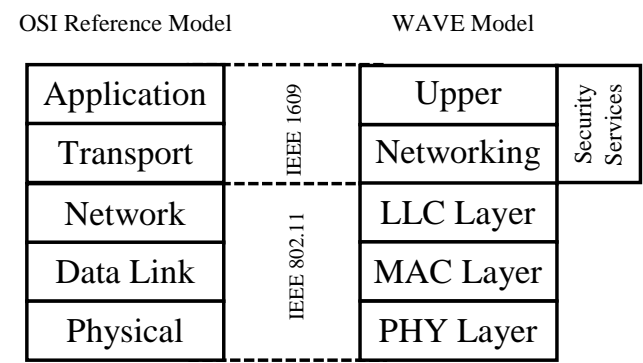

Fig.6. Relationship between OSI and WAVE reference model

The inclusion of OSI layer as well as the implementation of IEEE 802 architecture at the physical layer is necessary to properly establish a consistent protocol that can be universally adopted for the usage of VANET research. However, only lower layers are considered and implemented in the simulation. The reason is because of the data security, authentication of valid user and error checking on the upper layer is not included in the scope of the research. Besides, the interest of the study is to investigate and determine the behaviour of the developed VANET protocol with high mobility nodes on such a rapid change environment simulated using SUMO.

\section{COUPLED SIMULATION}

The interaction between the traffic and the network simulation is not considered in most researches. Typically, the traffic demands like vehicle's travelling details such as speed, direction and position along the time are generated offline in most cases to speed up the network simulation performance and the generated traffic information is reusable. However, one of the major flaws of using offline traffic data is that the road traffic can influence the network traffic but not vice versa. For instance, traffic congestion, accident information or hazard warning are influencing the driver's behaviour, where the information can be transmitted over the VANET network. Upon the information received, the driver should react by accelerating, decelerating or changing into another lane. This requires cooperation between the network and traffic simulation.

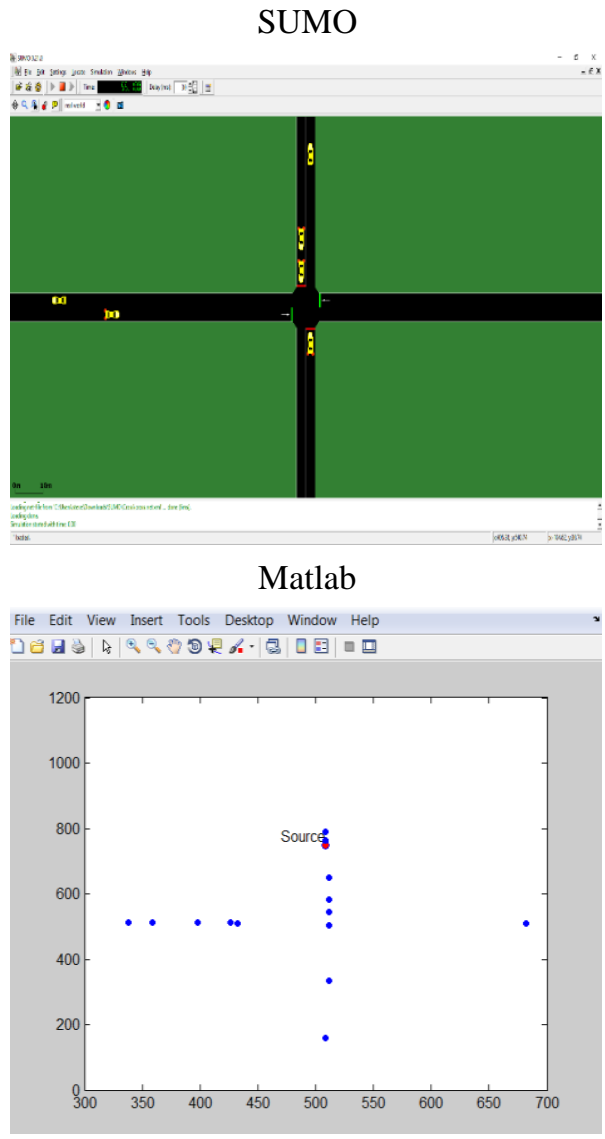

Fig.7. Coupling simulation between SUMO and Matlab

Many bidirectional coupled simulators have been developed and proposed for such cooperation, providing a detail insight on the impacts of network simulation. The hybrid simulation framework TraCI, a short term for Traffic Control Interface has been proposed, providing bidirectional coupling of network and road traffic simulation based on the MATLAB and SUMO frameworks, respectively. It acts as the interface between MATLAB and SUMO, and retrieves the values of simulated object while manipulating the traffic data online. The Fig.7 shows an example of coupling simulation between SUMO and MATLAB. The location of each vehicles generated in SUMO is transferred to MATLAB. The location received is then plotted in MATLAB.

An event-driven simulation technique is used in designing transmission protocol for VANET and it is also developed in the same platform, which is Matlab. An event can be defined as a change in state or activity of an action. Each event will only operates at a particular instant in time which indicates the change 
of state in the simulation. An event will be initiated to represent an action of a node in VANET. The Table. 1 shows the event structure for VANET simulation.

As described earlier, packet is passed from the top layer of OSI model in transmitter to the bottom layer and then up from bottom layer to the top on the receiver. Event type is used to indicate the direction of the packet and the OSI layer where the packet will be executed.

The instant in the event denotes the current simulation time for the action of the node. The instant described in event is important because it will be used in evaluating the performance of the network later in the research. Node identity in the event structure identifies which node that the action is being applied, while the network route stores the path of the packet transmission.

Table.1. Event structure

\begin{tabular}{|c|c|}
\hline Event parameter & Description \\
\hline Type & $\begin{array}{c}\text { Stage in OSI model such network layer, } \\
\text { physical layer }\end{array}$ \\
\hline Instant & Time of a particular event \\
\hline Node & Identity of the current node \\
\hline Route & Path from the source to the destination \\
\hline Packet detail & $\begin{array}{c}\text { Packet size, identity of next hop, and } \\
\text { identity of current hop }\end{array}$ \\
\hline
\end{tabular}

Lastly, the packet detail in the event containing the packet size, identity of next hop and identity of current node, which is used to determine the intended packet and intervene the intended packet from being broadcasted. The Fig. 8 illustrates the broadcast nature of a wireless vehicular node sending a packet to another node.
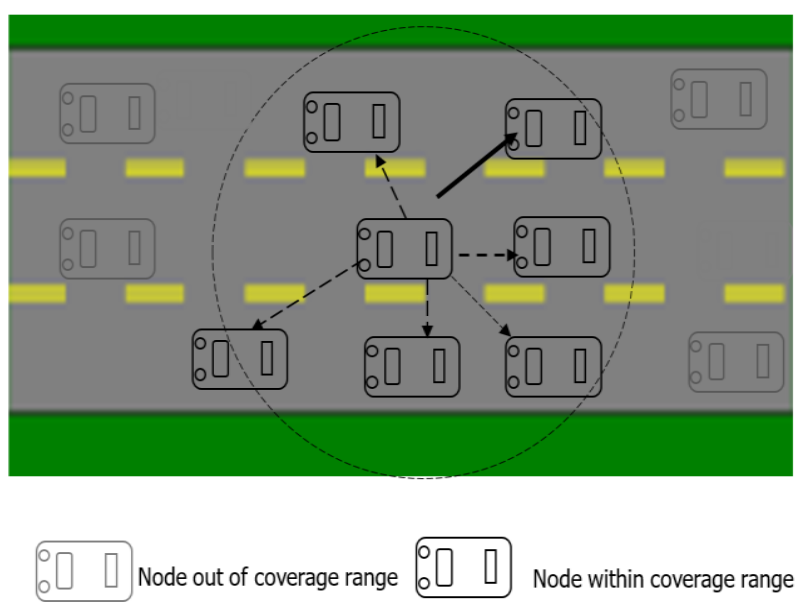

Fig.8. Broadcast nature of vehicular node

In VANET, DSRC is used to provide wireless communication between vehicles. As illustrated in Fig.8, a single transmission of a node could be received by all its neighbours that are within its transmission range. These phenomenon happened because of the broadcast nature of the radio communication.

After initialisation of network topology in SUMO, first event will be scheduled based on the initialised parameters and scenario given. After the event completed at the specified interval, the event will be updated with another event with new parameters based on the outcomes of the last event. The Fig.9 shows the event-driven based modelling process flowchart for simulation.

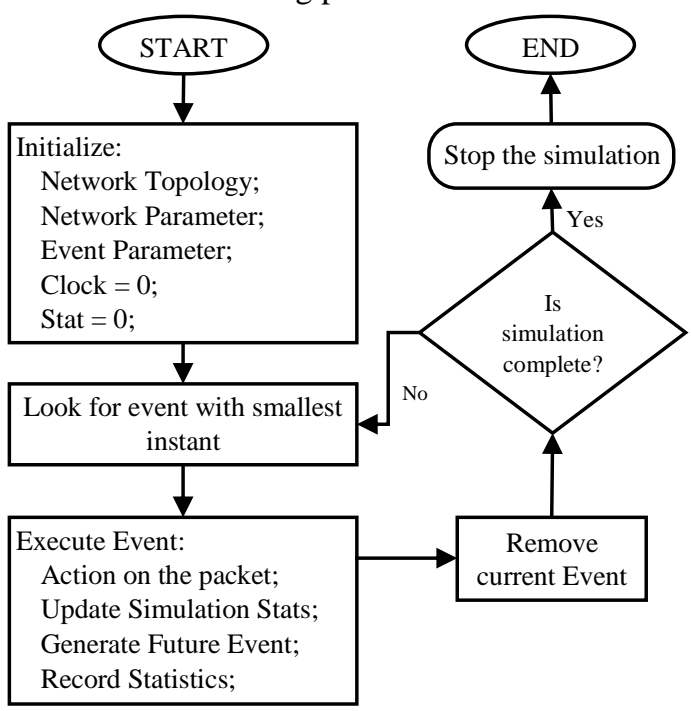

Fig.9. Flow chart of event-driven based modelling for simulation

As mentioned in previous section, event is created based on the given initialised parameters and scenario. Information such packet event, identity of source and destination, and packet size will be initialised and stored in the event before the packet passed to the upper layer in OSI model to determine the route availability from the source to the destination only. Other data conversion, security, authentication of valid user and error checking on the upper layer of OSI model are not included in this research.

\section{VEHICULAR AD HOC NETWORK SIMULATION}

As described in earlier section, SUMO is used to generate realistic vehicular mobility traces for VANET, which can be used for the simulation of vehicle-to-vehicle communication and the evaluation of related applications. The generated mobility traces are used to provide vehicular information such as speed, direction, arrival and departure time and location of each node over time. In this research, such traces are transferred and recorded in Matlab to create required components for VANET simulation such as details structure of all nodes, transmit and receive protocols, data traffic transmission and channels. The network simulation is developed in Matlab and the performance of the developed protocol will be evaluated in the same platform.

The traffic simulation of VANET is simulated in SUMO while the communication protocol of VANET is modelled in MATLAB by referring to the WAVE model defined by IEEE 802 group. Pseudo code for the transmission and NC protocol and its performance will be developed and evaluated in MATLAB mfile. The road layout in Jalan Sulaiman, Kota Kinabalu, Malaysia is imported to SUMO from OSM database. The imported map covers an area of approximately $100 \mathrm{~km}^{2}$. The generated traffic information in SUMO is then exported to the network simulator, which is MATLAB in this case. In the simulation, three mobile nodes are generated and travelling at the same speed of 30 $\mathrm{km} /$ hour towards the same direction as shown in Fig.10. Node 1 and node 2 wanted to communicate with each other but separated 
by a distance greater than their transmission range. Hence, the intermediate node 3 , which is located between node 1 and node 3 will act as a relay node to forward the packets.

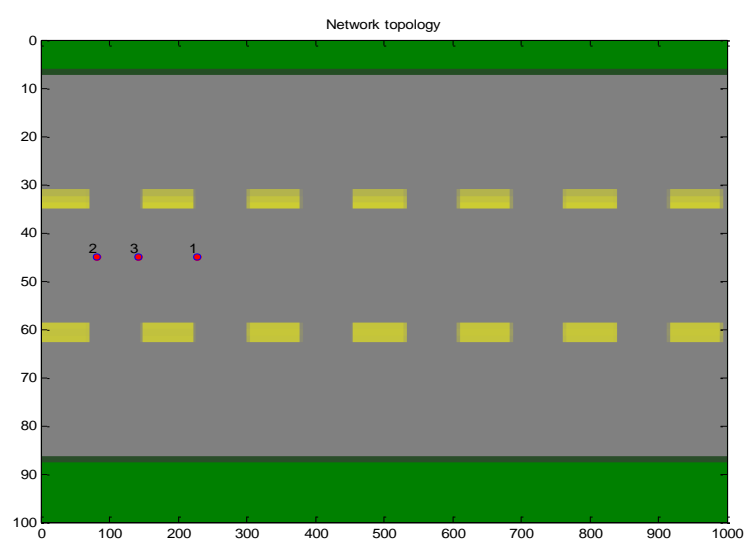

Fig.10. Simulation network topology

The Table. 2 shows the parameters and configuration used in the simulation. The vehicular nodes are assumed to communicate in a noiseless environment and each node has a transmission range up to $100 \mathrm{~m}$. Considering the scenario in Fig. 10, where node 1 has 500 packets to be delivered to node 3 before carrying to node 2 . The size of each packet is 1000 bit.

Table.2. Simulation setup

\begin{tabular}{|c|c|}
\hline Parameter & Value \\
\hline Network size & $1000 \times 100 \mathrm{~m}^{2}$ \\
\hline Number of vehicular node & 3 \\
\hline Transmission range & $100 \mathrm{~m}$ \\
\hline Packet quantity & 500 \\
\hline Packet Size & 1000 bit \\
\hline
\end{tabular}

\section{ASSESMENT AND EVALUATION}

In a simple wireless broadcast, the messages are transmitted by conventional store-and-forward technique with one packet per transmission. Unlike traditional store-and-forward method, the network coding (NC) could offer multiple contents in a single transmission, improving the network throughput using the bitwise exclusive or (XOR) operator. XOR operator is known as the anticoincidence operator or inequality detector as it provides a logic gate with equal inputs will produce a false output and a true output for the unequal inputs. One of the advantages of using XOR in $\mathrm{NC}$ is the encoded or combined packet will be remained in the same length as its original packet. The Fig.11 shows the example of encoding and decoding between two packets using XOR logical operator. As shown in the Fig. 11 below, the 8 bit packet from node A will combine with another 8 bit packet from node B. The size of the combined packet remains the same as the original packet from node A and B, as shown in Fig.11 below. Finally, both node A and B can decode the combined packet by XOR the packet with their own original non-coded packet.

\section{Encode

A \begin{tabular}{|c|c|c|c|c|c|c|c|c|}
\hline 1 & 1 & 1 & 0 & 0 & 1 & 1 & 0 \\
\hline
\end{tabular}
\begin{tabular}{|c|c|c|c|c|c|c|c|c|c|}
\hline B & 1 & 0 & 1 & 1 & 1 & 0 & 0 & 0 \\
\hline$A \oplus B$ & 0 & 1 & 0 & 1 & 1 & 1 & 1 & 0 \\
\hline
\end{tabular}

Decode $A$ from $A \oplus B$
$\left.\begin{array}{|c|c|c|c|c|c|c|c|c|c|c|}\hline A \oplus B & 0 & 1 & 0 & 1 & 1 & 1 & 1 & 0 \\
\hline \\
\hline\end{array}\right]$\begin{tabular}{c|c|c|c|c|c|c|c|c|c|}
\hline & 1 & 0 & 1 & 1 & 1 & 0 & 0 & 0 \\
\hline$A$ & 1 & 1 & 1 & 0 & 0 & 1 & 1 & 0 \\
\hline
\end{tabular}

Decode $B$ from $A \oplus B$

\begin{tabular}{|c|c|c|c|c|c|c|c|}
\hline & & & 0 & 1 & 0 & 1 & 1 \\
\hline & A & 1 & 1 & 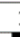 & & 1 & 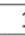 \\
\hline B & 1 & 0 & 1 & 1 & 1 & 0 & 0 \\
\hline
\end{tabular}

Fig.11. Encode and decode of XOR operator

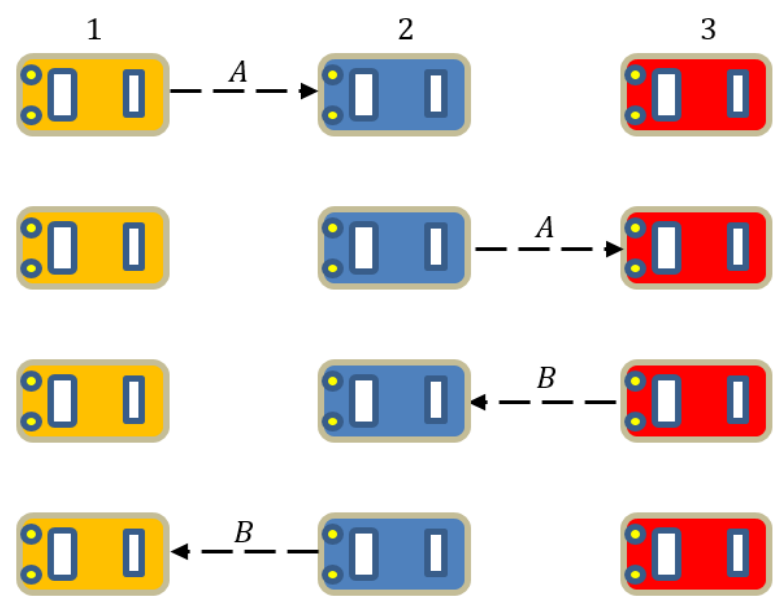

Fig.12. Store-and-forward based transmission

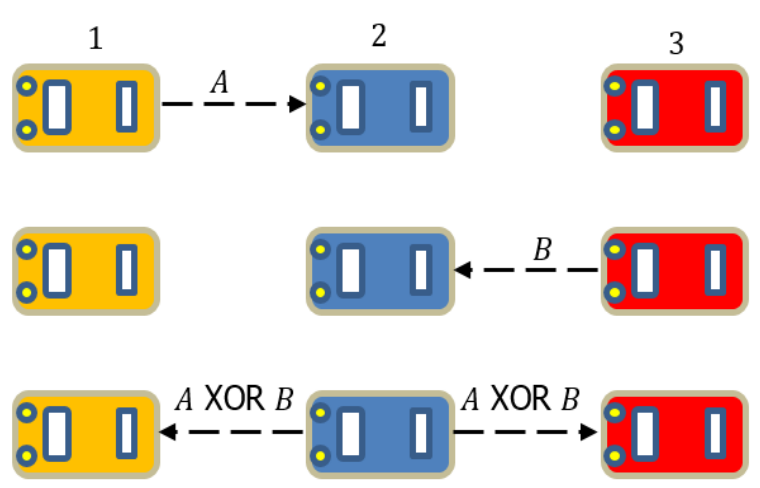

Fig.13. Network coding based transmission

Consider a simple wireless network as shown in Fig.12, node 1 has a packet intended for node 3 and node 3 also has a packet destined to node 2. However, both nodes are situated far apart from each other. Therefore, node 3 acts as an intermediate node to relay the packets from the source to the destination node. In a conventional store-and-forward approach as illustrated in Fig.12, node 1 send its packet to node 2 and then let node 2 forward the 
packet further to node 3 . Similarly, node 3 also send its packet to node 1 via node 2 . Overall, the total number of transmission required to complete the packet exchanges between node 1 and node 2 is equal to four transmissions.

In contrast to the transmission using $\mathrm{NC}$, the packet exchanges between node 1 and 3 can be accomplished with fewer transmission than the conventional store-and-forward method. First, node 1 transmits its packet to node 2. Second, node 3 also sends its packet to node 2 . Node 2 combines the received packets into packet A XOR B and then broadcasts the XORed packet to both node 1 and 3 . Finally, node 1 and node 3 decode their received message with $\mathrm{XOR}$ operation to obtain the original message. In total, NC only requires three transmission time slots to deliver the packets to its respective destination node as shown in Fig.13.

The following figures showed the comparison of the total time required for transmission all available messages, average throughput and energy consumption between $\mathrm{NC}$ and the conventional store-and-forward transmission protocol.

First, the comparison of the total time required for transmission all available messages between $\mathrm{NC}$ and the conventional store-and-forward transmission protocol is presented in Fig.14. The simulation result shows the total time to transmit all the data using $\mathrm{NC}$ is fewer than the conventional store-and-forward transmission protocol. NC requires 1.205 second to transmit all the 500 packets, whereas store-and-forward uses 1.655 to transmit all packet to destination. The total number of transferred bit in the overall network is $100 \mathrm{kbits}$, where both node 1 and node 2 received 500 packets $\times 1000$ bits $=50$ kbits each. NC based network shows an improvement of $27.2 \%$ over the store-and-forward based network. As shown in Fig.14, the improvement of the network begins at 0.0476 seconds where the first coding occurred in the network. The NC based network is perform better than the store-and-forward based network because $\mathrm{NC}$ able to perform coding in the intermediate node by combining the packets queued in the buffer and transmit the coded packet to their destinations simultaneously. Unlike NC, store-and-forward will queue the packet in the node buffer and transmit the packet individually to their respective destination based on First-in-firstout (FIFO) principle, hence it consume more time slots to deliver the same amount of packets as in NC network.

The Fig. 15 shows the comparison of the average throughput over time between NC based network and conventional store-andforward network. The average throughput is the average rate of successful message delivery over the communication channel. The simulation result shows the $\mathrm{NC}$ has higher average throughput than the store-and-forward, which means the NC has high average rate of successful packet delivery. In the early transmission, the average throughput in $\mathrm{NC}$ based network is noise-like as depicted in Fig. 15. This happened because the NC network suffer from the inconsistency in packet delivery due to inconsistent in transmission deferral caused by the random backoff time introduced in the network. The random backoff procedure is employed in the network to defer the transmission for a short time to wait for the channel to be idle before resume back to the packet transmission.

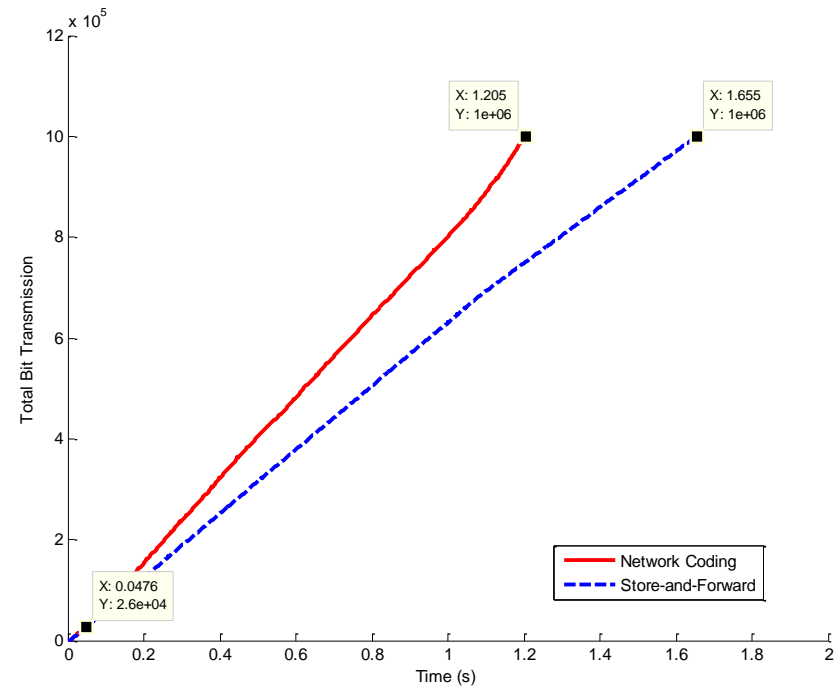

Fig.14. Simulation results of total number of transferred bits over time

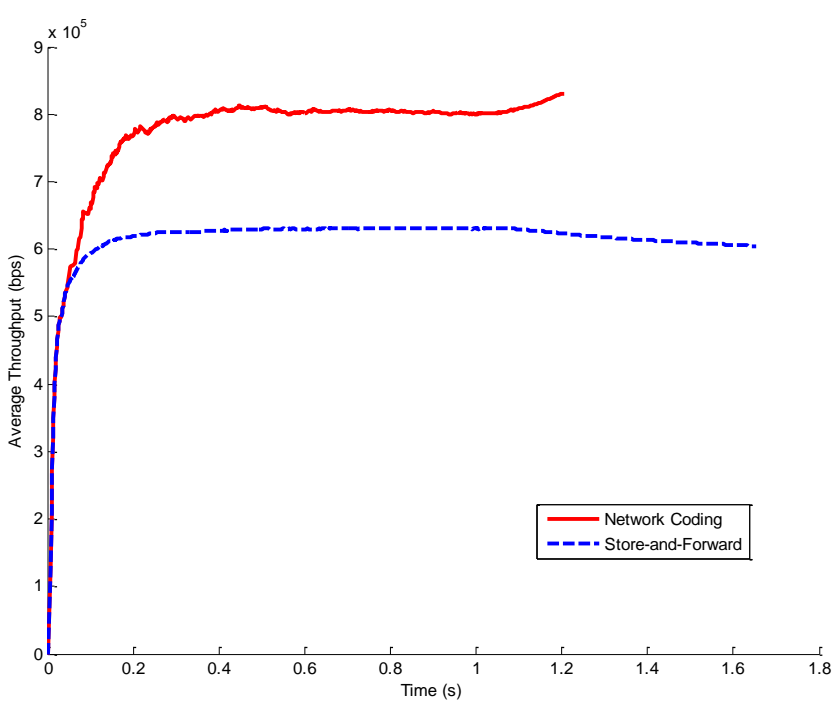

Fig.15. Simulation results of average throughput over time

The Fig.16 presents the simulation result of energy consumed for packet transmission in the network. The simulation shows that the energy consumption for NC based network is $0.1026 \mathrm{~J}$ which is much lesser than the store-and-forward based network with $0.12 \mathrm{~J}$. The total energy saved from NC approach is about $0.017 \mathrm{~J}$, which can be used to make another 84 transmissions based on NC approach. Therefore, it can be concluded that NC method not only able to increase the network throughput, but also decreases the energy usage in the network. This phenomenon happened because the less transmission used in $\mathrm{NC}$ leads to less efforts required to deliver the packet as compared to the store-and-forward. Though, the NC based network shows improvement of throughput and energy consumption over store-and-forward. But the chances to perform NC in wireless network is unlikely if the packet is not transmitted to the potential NC node. Hence, routing protocol is proposed in this work to increase the chances to perform $\mathrm{NC}$ in wireless node. 


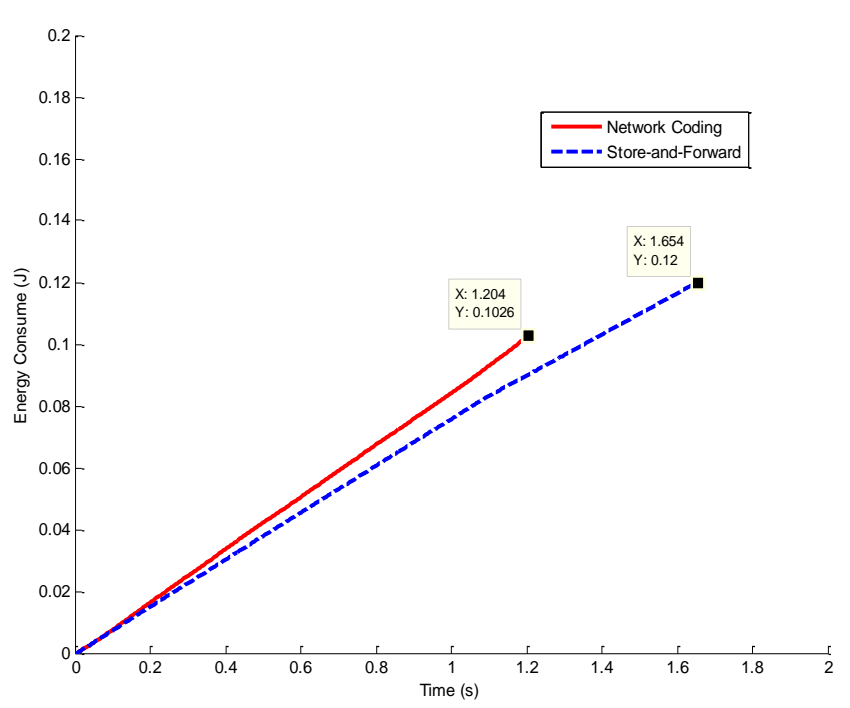

Fig.16. Simulation results of energy consume over time

\section{CONCLUSION}

Vehicular ad hoc network (known as VANET) has been gaining popularity and attention from both research and industry communities in recent years due to its inexpensive, flexibility, fault tolerance and ability to function in high mobility pattern and rapid changing topology. However, a feasible and repeatable simulation network is necessary in order to evaluate and assess the developed VANET protocol and recognise its potential impacts on the realistic environment to reduce the high deployment cost.

The bidirectional simulation between traffic and network have been developed in this work. SUMO, an open source, multi-modal traffic simulator is employed in this research to generate traffic information including vehicle's travelling details such as speed, direction and position along the time.

Whereas, open system interconnection (OSI) model with wireless access vehicular environment (WAVE) parameters defined in IEEE 802 group has been modelled in MATLAB as the study model. Several assumptions have been made to reduce the complexity without compromising the overall accuracy of the developed model.

Many studies on VANET have been done but the works are rather rudimentary because of simplistic assumptions in designing topology which leads researchers to inaccurate conclusion. In this research, the vehicular model is constructed and edited using existing traffic information. In order to observe the performance of the developed model, traffic flow is generated randomly using mobility model built in SUMO. Still, simulation of this research is not thorough to reflect the real-world vehicle mobility as the behaviors of vehicle like over-taking and sudden braking are not considered in this work. This is crucial because vehicular traffic pattern can greatly affect the node connectivity and link establishment. Any sudden link breakage and connection loss will lead to degradation of the entire network delivery.

Besides, several assumptions have been made in wireless communication network simulation where the communication link between the nodes is assumed to be ideal and noiseless. Thus, the packet will reach the next hop without any interference. But, in real world, the quality of link between a pair of node is different and could be affected by the transmission power and sensitivity. It is also assumed that the transmission range is homogenous where every node in the network has the same transmission range. In fact, different transmission power will determine different transmission ranges. Moreover, in real implementation, heterogeneous wireless network hardware with different sensitivity are often adopted, resulting in different transmission ranges. Therefore, in order for the developed model to be used in lossy network, the developed model can be extended by considering the link quality, directional and range of transmission of the wireless network. Therefore, the future work is motivated to improve the overall functions of VANET by developing a realistic VANET model that could represent the target system.

\section{ACKNOWLEDGMENT}

The authors would like to acknowledge the financial assistance of the Ministry of Education Malaysia (KPM) under Exploratory Research Grant Schemes (ERGS), grant no. UMS/ERG0046-ICT-1/2013, and Fundamental Research Grant Schemes (FRGS), grant no. UMS/FRG0365-ICT-1/2014.

\section{REFERENCES}

[1] M. Seredynski, G. Danoy, M. Tabatabaei, P. Bouvry and Y. Pigne, "Generation of Realistic Mobility for VANETs using Genetic Algorithms", Proceedings of IEEE Congress on Evolutionary Computation, pp. 334-337, 2012.

[2] F. Cunha, L. Villas, A. Boukerche, G. Maia, A. Viana, R.A.F. Mini and A.A.F. Loureiro, "Data Communication in VANETs: Protocols, Applications and Challenges", Ad Hoc Networks, Vol. 44, pp. 90-103, 2016.

[3] P. Vijayakumar, M. Azees, A. Kannan and L.J. Deborah, "Dual Authentication and Key Management Techniques for Secure Data Transmission in Vehicular Ad Hoc Network", IEEE Transactions on Intelligent Transportation Systems, Vol. 17, No. 4, pp. 1015-1028, 2016.

[4] S. Djahel, R. Doolan, G.M. Muntean and J. Murphy, "A Communications-Oriented Perspective on Traffic Management Systems for Smart Cities: Challenges and Innovative Approaches", IEEE Communications Surveys and Tutorials, Vol. 17, No. 1, pp. 125-151, 2015.

[5] K.T.K. Teo, R.K.Y. Chin, S.E. Tan, C.H. Lee and K.G. Lim, "Performance Analysis of Enhanced Genetic Algorithm based Network Coding in Wireless Networks", Proceedings of $8^{\text {th }}$ Asia Modelling Symposium, pp. 213-218, 2014.

[6] S.E. Tan, H.T. Yew, M.S. Arifianto, I. Saad and K.T.K. Teo, "Queue Management for Network Coding in Ad Hoc Networks", Proceedings of $3^{\text {rd }}$ International Conference on Intelligent Systems Modelling and Simulation, pp. 657-662, 2012.

[7] P. Kumar, R. Merzouki, B. Conrard and V. Coelen, "Multilevel Modeling of the Traffic Dynamic", IEEE Transactions on Intelligent Transportation Systems, Vol. 15, No. 3, pp. 1066-1082, 2014.

[8] T. Mantoro and M. Reza, "Performance analysis of AODV and DSDV using SUMO, MOVE and NS2", Proceedings of International Conference on Informatics and Computing, pp. 372-376, 2016. 
[9] D.F. Allan and A.M. Farid, "A Benchmark Analysis of Open Source Transportation-Electrification Simulation Tools", Proceedings of $18^{\text {th }}$ IEEE International Conference on Intelligent Transportation Systems, pp. 1202-1208, 2015.

[10] A.K.H Souley and S. Cherkaoui, "Advanced Mobility Models for Ad Hoc Network Simulations", Proceedings of IEEE International Conference on Systems Communications, pp. 50-55, 2015.

[11] Y.S. Chia, Z.W. Siew, H.T. Yew, S.S. Yang and K.T.K. Teo, "An Evolutionary Algorithm for Channel Assignment Problem in Wireless Mobile Networks", ICTACT Journal on Communication Technology, Vol. 3, No. 4, pp. 613-618, 2012.

[12] S.E. Tan, Z.W. Siew, Y.K. Chin, S.C.K. Lye and K.T.K. Teo, "Minimizing Network Coding Nodes in Multicast Tree Construction Via Genetic Algorithm", Proceedings of $4^{\text {th }}$
International Conference on Computational Intelligence, Communication Systems and Networks, pp. 399-404, 2012.

[13] C.C. Lin, L.C. Hu, K.H. Hsu and H.H. Li, "On the Performance of Vehicular Group Communications in IEEE 1609/802.11(p) Networks", Proceedings of IEEE Vehicular Technology Conference, pp. 1-5, 2011.

[14] K.A. Rahman and K.E. Tepe, "Towards A Cross-Layer Based Mac for Smooth V2V and V2I Communications for Safety Applications in DSRC/Wave Based Systems", Proceedings of IEEE Symposium on Intelligent Vehicles, pp. 969-973, 2014.

[15] K. Bilstrup, E. Uhlemann, E.G. Strom and U. Bilstrup, "Evaluation of the IEEE 802.11p MAC Method for Vehicleto-Vehicle Communication", Proceedings of International Conference on Vehicular Technology, pp. 1-5, 2008. 